Pacific Journal of Mathematics

EMBEDDED EIGENVALUES AND VIRTUAL POLES 


\title{
EMBEDDED EIGENVALUES AND VIRTUAL POLES
}

\section{JAMES S. HowLAND}

\begin{abstract}
An embedded eigenvalue of simple multiplicity is perturbed by an operator of finite rank. The resulting spectral concentration is shown to be due to the pole of the continuation of a certain analytic function.
\end{abstract}

If $\lambda_{0}$ is point eigenvalue of the selfadjoint operator $T_{0}$, then it may happen that $\lambda_{0}$ "disappears" under perturbation in the sense that the perturbed operator $T_{\varepsilon}=T_{0}+\varepsilon V$ has a purely continuous spectrum near $\lambda_{0}$ for arbitrarily small positive $\varepsilon$. As McLeod [10] remarks, there are two parts of the theory of this phenomenon. There is, first of all, the theory of spectral concentration, in which it is shown that as $\varepsilon \rightarrow 0+$ there is an asymptotic "concentration" of the spectral measure of $T_{\varepsilon}$ near $\lambda_{0}$. Spectral concentration has been discussed for isolated eigenvalues by many authors (see [1], [10], and [8, Chap. VIII]), and for eigenvalues embedded in the continuous spectrum by Friedrichs [4] and the present author [5]. Secondly, there is the conjecture, verified in certain examples (see [2], [10]), that this concentration of the spectral measure is due to a virtual pole of the resolvent $R(z, \varepsilon)=\left(T_{\varepsilon}-z\right)^{-1}$. For example, if $T_{\varepsilon}$ is a differential operator and $R(z, \varepsilon)$ an integral operator with Green's function kernel $g_{\varepsilon}(x, y ; z)$, this means that $g_{\varepsilon}$ can be continued meromorphicly from $\operatorname{Im} z>0$ to a neighborhood of $\lambda_{0}$ (on which the continuation will not agree with the values of $g_{\varepsilon}(x, y ; z)$ for $\left.\operatorname{Im} z<0\right)$, and that this continuation will have, slightly below the axis, a pole $\zeta(\varepsilon)$ which tends to $\lambda_{0}$ as $\varepsilon \rightarrow 0+$, and which accounts for the concentration in much the same way that a pole on the axis might account for a point eigenvalue. This example defines the problem-in a more general situation, the first step toward a solution is to find a replacement for the Green's function which can be continued meromorphicly.

In [5], the author considered spectral concentration of embedded eigenvalues of simple multiplicity, under perturbations of finite rank. In the present paper, we shall discuss virtual poles in the context. The quantity chosen to replace the Green's function is the inverse Weinstein-Aronszajn matrix; that is to say, the inverse of the matrix of the restriction of $I+\varepsilon V\left(T_{0}-z\right)^{-1}$ to the (finite dimensional) range of the perturbation $V$ (cf. [8, Chap. IV, §6]).

The organization of the paper is as follows. In $\S 1$, we state the basic assumptions and note a formula (Lemma 1.1) which is fundamental to our approach. The formulation of the problem is similar to that of Kuroda [9]. The existence of a virtual pole $\zeta(\varepsilon)$ is then 
proved for sufficiently small $\varepsilon$ in $\S 2$. The spectral theory of $T_{\varepsilon}$ is discussed in $\S 4$, where it is shown that $T_{\varepsilon}$ has an eigenvalue near $\lambda_{0}$ if and only if $\zeta(\varepsilon)$ is real, in which case the eigenvalue is $\zeta(\varepsilon)$ itself. Section 3 contains a general result on continuations of Stieltjes transforms which is used in $\S 4$. Next, we consider the asymptotic spectral theory of $T_{\varepsilon}$ as $\varepsilon \rightarrow 0+$, in the case that $\zeta(\varepsilon)$ is not real. In $\S 5$, we obtain an asymptotic formula on the range of $V$ for the spectral density near $\lambda_{0}$, which corresponds to the formula of Wigner and Weiskopff mentioned by Friedrichs [4]. This formula is used in $\S 6$ to prove a result on spectral concentration near $\lambda_{0}$. Finally, in $\S 7$, we discuss, under certain conditions of nondegeneracy, the relationship between $\zeta(\varepsilon)$ and the formal perturbation series for the perturbed eigenvalue, obtaining in this case an asymptotic formula for the imaginary part of $\zeta(\varepsilon)$. Section 8 discusses three examples.

1. Assumptions and preliminary facts. Let $T_{0}$ be a self-adjoint operator on a Hilbert space $\mathscr{H}$ with domain $\mathscr{D}\left(T_{0}\right)$, and $V$ a bounded self-adjoint operator of finite rank $r . V$ may then be written in the form $V=\sum_{i=1}^{r} c_{i}\left(\cdot, \varphi_{i}\right) \varphi_{i}$ where $c_{1}, \cdots, c_{r}$ are nonvanishing real numbers and $\left\{\varphi_{1}, \cdots, \varphi_{r}\right\}$ is a finite orthonormal set spanning the range $\mathscr{R}(V)$ of $V$. If $\varepsilon>0$, then the operator $T_{\varepsilon}=T_{0}+\varepsilon V$ with $\mathscr{D}\left(T_{\varepsilon}\right)=$ $\mathscr{D}\left(T_{0}\right)$ is also self-adjoint. Let

$$
R_{0}(z)=\left(T_{0}-z\right)^{-1} \text { and } R(z, \varepsilon)=\left(T_{\varepsilon}-z\right)^{-1}
$$

be the resolvents of $T_{0}$ and $T_{\varepsilon}$, and $E_{0}(\lambda)$ and $E_{\varepsilon}(\lambda)$ the corresponding spectral resolutions. If $S$ is a Borel set, we define

$$
E_{\varepsilon}[S]=\int_{S} d E_{\varepsilon}(\lambda)
$$

for $\varepsilon \geqq 0$.

The following assumption will be made throughout the paper, and is an implicit hypothesis of all theorems.

BAsic Hypothesis. Let $\lambda_{0}$ be an eigenvalue of $T_{0}$ of simple multiplicity and $\psi_{0}$ a corresponding normalized eigenvector. Let $P_{0}=$ $\left(\cdot, \psi_{0}\right) \psi_{0}$ and $P=I-P_{0}$. We shall assume that for $i, j=1, \cdots, r$ the analytic function $\left(R_{0}(z) P \varphi_{i}, \varphi_{j}\right)$ has an analytic continuation from the upper half-plane into a neighborhood of $\lambda_{0}$ in the complex plane.

In the sequel, we shall let $N=\left\{z:\left|z-\lambda_{0}\right| \leqq \delta_{0}\right\}$ be a fixed closed disc contained in the above neighborhoods for all $i, j=1, \cdots, r$. Since $\left[R_{0}(z)\right]^{*}=R_{0}(\bar{z})$, it is a consequence of the above assumption that $\left(R_{0}(z) P \varphi_{i}, \varphi_{j}\right)$ has a corresponding continuation from the lower half- 
plane to a neighborhood of $N$. These two continuations are in general not identical on $N$, since $\lambda_{0}$ need not be an isolated eigenvalue. In order to distinguish between them, we shall introduce the following notation: Let $F(z)$ be a function defined and analytic for $\operatorname{Im} z \neq 0$. The continuation of $F(z)$ from $\operatorname{Im} z>0$ to $N$ will be denoted by a subscript ' + ', while the continuation from $\operatorname{Im} z<0$ to $N$ will be denoted by a subscript ' - '. For example, $\left(R_{0}(z) \varphi_{i}, \varphi_{j}\right)_{+}$denotes the continuation from $\operatorname{Im} z>0$. Similarly, we shall encounter such notations as $W_{ \pm}(z, \varepsilon)$ and $\left[W_{a}(z, \varepsilon)\right]_{ \pm}$.

The matrix whose elements are $a_{i j}, i, j=1, \cdots, r$, will be denoted by mat $\left\{a_{i j}\right\}$.

For $\operatorname{Im} z \neq 0$, let $W(z, \varepsilon)$ be the restriction of $I+\varepsilon V R_{0}(z)$ to $\mathscr{R}(V)$. Since $\mathscr{R}(V)$ is invariant under $I+\varepsilon V R_{0}(z), W(z, \varepsilon)$ is an operator on the finite dimensional space $\mathscr{R}(V)$, and is easily seen to have the matrix

$$
\operatorname{mat}\left\{\delta_{i j}+\varepsilon c_{j}\left(R_{0}(z) \varphi_{i}, \varphi_{j}\right)\right\}
$$

with respect to the basis $\varphi_{1}, \cdots, \varphi_{r}$. Moreover, $W^{-1}(z, \varepsilon)$ exists for $\operatorname{Im} z \neq 0$ and every $\varepsilon \geqq 0$, and is the restriction to $\mathscr{R}(V)$ of the operator $I-\varepsilon V R(z, \varepsilon)$ (see $[5, \S 1]$ or [10]). We also define $W_{a}(z, \varepsilon)$ to be the restriction to $\mathscr{R}(V)$ of $I+\varepsilon V R_{0}(z) P$, so that

$$
W(z, \varepsilon)=W_{a}(z, \varepsilon)+\left(\lambda_{0}-z\right)^{-1} V P_{0} .
$$

In analogy with $(1.1), W_{a}(z, \varepsilon)$ has the matrix

$$
\operatorname{mat}\left\{\delta_{i j}+\varepsilon c_{j}\left(R_{0}(z) P \varphi_{i}, \varphi_{j}\right)\right\} \text {. }
$$

The following formula for $W^{-1}(z, \varepsilon)$ is basic to our approach.

Lemma 1.1. $W_{a}^{-1}(z, \varepsilon)$ exists for $\operatorname{Im} z \neq 0$ and

$$
W^{-1}(z, \varepsilon)=W_{a}^{-1}(z, \varepsilon)\left[I-\Delta(z, \varepsilon)\left(W_{a}^{-1}(z, \varepsilon) \cdot, \psi_{0}\right) V \psi_{0}\right]
$$

where

$$
\Delta(z, \varepsilon)=\varepsilon\left[\lambda_{0}-z+\varepsilon\left(W_{a}^{-1}(z, \varepsilon) V \psi_{0}, \psi_{0}\right)\right]^{-1} .
$$

For the proof, see [5, $\S 1$, Lemma 1.1].

Proposition. Under the Basic Hypothesis, the following hold for all sufficiently small positive $\varepsilon$ :

(a) $W_{a}(z, \varepsilon)$ and $W_{a}^{-1}(z, \varepsilon)$ have analytic continuations to a neighborhood of $N$ from both half-planes. For a suitably small $\varepsilon_{0}>0$, these continuations, $\left[W_{a}(z, \varepsilon)\right]_{ \pm}$and $\left[W_{a}^{-1}(z, \varepsilon)\right]_{ \pm}$are bounded on $N$, uniformly for $0 \leqq \varepsilon \leqq \varepsilon_{0}$. 
(b) If $V P_{0} \neq 0$, then $W(z, \varepsilon), W^{-1}(z, \varepsilon)$, and $\Delta(z, \varepsilon)$ have meromorphic continuations to a neighborhood of $N$ from both half-planes. $W_{ \pm}(z, \varepsilon)$ is analytic on $N$ with the exception of a simple pole at $\lambda_{0}$. The poles of $W_{ \pm}^{-1}(z, \varepsilon)$ coincide with those of $\Delta_{ \pm}(z, \varepsilon)$. The residues at these poles have rank one.

Proof. Existence and uniform boundedness of the continuations $\left[W_{a}(z, \varepsilon)\right]_{ \pm}$is immediate from the Basic Hypothesis and (1.3). The Neumann series expansion of $W_{a}^{-1}(z, \varepsilon)$ in powers of $\varepsilon$ (cf. [5; $\left.\S 3\right]$ ) converges uniformly on $N$, so that the rest of (a) follows. (b) follows easily from (a) and an inspection of formulae (1.2), (1.4) and (1.5).

2. Virtual poles. We are now able to prove the existence of a virtual pole of $W^{-1}(z, \varepsilon)$.

THEOREM 1. Let $V P_{0} \neq 0$. Then for all sufficiently small positive $\varepsilon, W_{+}^{-1}(z, \varepsilon)$ has a unique pole $\zeta(\varepsilon)$ in $N . \zeta(\varepsilon)$ is a simple pole with residue of rank one, $\operatorname{Im} \zeta(\varepsilon) \leqq 0$ and $\zeta(\varepsilon) \rightarrow \lambda_{0}$ as $\varepsilon \rightarrow 0+$. Moreover, $\zeta(\varepsilon)$ is a complex analytic function of $\varepsilon$ at $\varepsilon=0$.

Proof. By the Proposition of $\S 1$, it suffices to consider solutions of the equation

$$
\varepsilon \Delta_{+}^{-1}(z, \varepsilon)=\lambda_{0}-z+\varepsilon\left(W_{a}^{-1}(z, \varepsilon) V \psi_{0}, \psi_{0}\right)+=0 .
$$

Let $0<\rho \leqq \rho_{0}$. Then since $\left(W_{a}^{-1}(z, \varepsilon) V \psi_{0}, \psi_{0}\right)_{+}$is bounded on $N$ uniformly in $\varepsilon, \varepsilon \Delta_{+}^{-1}(z, \varepsilon)-\left(\lambda_{0}-z\right)$ can be made arbitrarily small uniformly on $\left|z-\lambda_{0}\right|=\rho$, by choosing $\varepsilon$ sufficiently small. By Rouche's Theorem, $\varepsilon \Delta_{+}^{-1}(z, \varepsilon)$ and $\left(\lambda_{0}-z\right)$ have the same number of zeros (counting multiplicities) inside $\left|z-\lambda_{0}\right|=\rho$. Choosing $\rho=\rho_{0}$ gives existence, uniqueness and simplicity of the pole $\zeta(\varepsilon)$ of $\Delta_{+}(z, \varepsilon)$ in $N$, while choosing $\rho$ arbitrarily small shows that $\zeta(\varepsilon) \rightarrow \lambda_{0}$ as $\varepsilon \rightarrow 0+$. Since the coefficient of $\Delta_{+}(z, \varepsilon)$ in (1.4) is of rank one, the residue of $W_{+}^{-1}(z, \varepsilon)$ has rank one. If $\operatorname{Im} z>0, W_{+}^{-1}(z, \varepsilon)$ is the restriction of $I-\varepsilon V R(z, \varepsilon)$ to $\mathscr{R}(V)$, and therefore has no pole. Hence, $\operatorname{Im} \zeta(\varepsilon) \leqq 0$. Analyticity of $\zeta(\varepsilon)$ follows from a general result [3; p. 101, Th. 7.1] on analyticity of inverse functions.

CoRollary. $W_{-}^{-1}(z, \varepsilon)$ has, for sufficiently small positive $\varepsilon$, the unique pole $\zeta_{-}(\varepsilon)=\bar{\zeta}(\varepsilon)$. As a complex analytic function of $\varepsilon, \zeta_{-}(\varepsilon)=\bar{\zeta}(\bar{\varepsilon})$.

Proof. We simply observe that for real $\varepsilon, \Delta_{-}(z, \varepsilon)=\bar{\Delta}_{+}(\bar{z}, \varepsilon)$ since $R_{0}(z)=R_{0}^{*}(\bar{z})$. The result follows easily. 
3. Continuations of Stieltjes transforms. The following simple result on meromorphic continuations of Stieltjes transforms of matrixvalued measures will be used in the next section.

THEOREM 2. Let $M$ be a positive Hermitian matrix-valued, finite Borel measure on $(-\infty,+\infty)$, and

$$
F(z)=\int_{-\infty}^{+\infty}(t-z)^{-1} d M(t) \quad \operatorname{Im} z \neq 0
$$

its Stieltjes transform. If $F(z)$ has a meromorphic continuation $F_{+}(z)$ from $\operatorname{Im} z>0$ to a disc $D$ about the origin, then to $D$.

(a) $F(z)$ has a meromorphic continuation $F_{-}(z)$ from $\operatorname{Im} z<0$

(b) The poles of $F_{-}(z)$ have nonnegative imaginary parts and are the reflections in the real axis of the poles of $F_{+}(z)$.

(c) If $F_{+}(z)$ has a simple pole at $z=0$, then $M$ has a point mass at the origin, equal to the residue of $F_{+}(z)$ at the origin.

Proof. From $\operatorname{Im} z \neq 0$, we have $F(z)=F^{*}(\bar{z})$, and hence by continuation

$$
F_{-}(z)=F_{+}^{*}(\bar{z})
$$

which proves (a) and (b). For (c), observe that if

$$
F_{+}(z)=(A / z)+\mathrm{O}(1)
$$

and

$$
F_{-}(z)=(B / z)+O(1)
$$

on $D$, then by $(3.1)$

$$
B=A^{*} \text {. }
$$

Now observe that for sufficiently small real $\lambda$, we have

$$
\begin{aligned}
M^{\prime}(\lambda) & =(1 / 2 \pi i)\left[F_{+}(\lambda)-F_{-}(\lambda)\right] \\
& =\left(A-A^{*}\right) /(2 \pi i \lambda)+\mathrm{O}(1)
\end{aligned}
$$

where $M^{\prime}(\lambda)$ is the spectral density for the absolutely continuous part of $M$. Since $M^{\prime}(\lambda)$ is a positive Hermitian matrix both for $\lambda>0$ and $\lambda<0$, we must have

$$
A=A^{*}=B \text {. }
$$

Now if $\delta>0$ is sufficiently small, it is clear that $M$ is absolutely continuous on $(-\delta,+\delta)$ with smooth derivative, except possibly at $z=0$. It is therefore easy to see that 


$$
\begin{aligned}
2 \pi i M(-\delta,+\delta) & =\int_{|z|=\delta} F(z) d z \\
& =\int_{|z|=\delta} F_{+}(z) d z+\int_{C}\left[F_{-}(z)-F_{+}(z)\right] d z
\end{aligned}
$$

where $C$ is the lower half of the (positively oriented) contour $|z|=\delta$. But the first term of the right side of (3.6) is equal to $2 \pi i A$, while the second is $\mathrm{O}(\delta)$, since its integrand is bounded on $C$ by $(3.2),(3.3)$ and (3.4). Hence,

$$
\lim _{\delta \rightarrow 0+} M(-\delta,+\delta)=A
$$

which proves the result.

4. Spectral theory. If $T=\int \lambda d E(\lambda)$ is self-adjoint and $S$ is a Borel subset of the reals, then the part of $T$ in $S$ is the operator $T E[S]$, considered as an operator on $E[S] \mathscr{H}$. $T$ is absolutely continuous on $S$ if and only if the part of $T$ in $S$ is absolutely continuous, or equivalently if and only if the measure $d(E(\lambda) E[S] x, x)$ is absolutely continuous with respect to Lebesgue measure for every $x$ in $\mathscr{Z}$.

If $\mathscr{C l}$ is the smallest subspace of $\mathscr{C}$ which contains $\varphi_{1}, \cdots, \varphi_{r}$ and reduces $T_{0}$, then $\mathscr{t}$ also reduces $T_{\varepsilon}$, and $T_{\varepsilon}=T_{0}$ on the orthogonal complement $\mathscr{C}^{\perp}$ of $\mathscr{C}$. For our purposes, it therefore suffices to assume that $\mathscr{C}=\mathscr{C}$ (see $[5,7,9])$. Let $G=\left(\lambda_{0}-\rho_{0}, \lambda_{0}+\rho_{0}\right)$ be the intersection of the interior of $N$ with the real axis.

Theorem 3. Assume that $\mathscr{C}=\mathscr{C l}$. Then $V P_{0} \neq 0$ and, for all sufficiently small positive $\varepsilon$,

(a) $T_{\varepsilon}$ has an eigenvalue in $G$ if and only if $\operatorname{Im} \zeta(\varepsilon)=0$, in which case $\zeta(\varepsilon)$ itself is the unique eigenvalue of $T_{\varepsilon}$ in $G$, and has simple multiplicity.

(b) The continuous part of $T_{\varepsilon}$ in $G$ is absolutely continuous and unitarily equivalent to the part of $T_{0}$ in $G \sim\left\{\lambda_{0}\right\}$.

Proof. Since

$$
W^{-1}(z, \varepsilon)=I-\varepsilon \operatorname{mat}\left\{c_{j}\left(R(z, \varepsilon) \varphi_{i}, \varphi_{j}\right)\right\}
$$

it follows that for $i, j=1, \cdots, r,\left(R(z, \varepsilon) \varphi_{i}, \varphi_{j}\right)$ is continuous on $G \sim$ $\{\zeta(\varepsilon)\}$, and hence that $d\left(E_{\varepsilon}(\lambda) \varphi_{i}, \varphi_{i}\right)$ is absolutely continuous on $G \sim\{\zeta(\varepsilon)\}$. Since $\mathscr{C}=\mathscr{C}$, it follows that $T_{\varepsilon}$ is absolutely continuous on $G \sim$ $\{\zeta(\varepsilon)\}$. (See [5], proof of Theorem 1). However, the absolutely continuous parts of $T_{0}$ and $T_{\varepsilon}$ are unitarily equivalent by the RosenblumKato Theorem [8; Th. 4.4, p. 540], so that part (b) follows. Since $G \sim\{\zeta(\varepsilon)\}=G$ when $\operatorname{Im} \zeta(\varepsilon)<0$, it remains only to show that $\zeta(\varepsilon)$ 
is an eigenvalue of simple multiplicity whenever $\operatorname{Im} \zeta(\varepsilon)=0$. Observe that the Stieltjes transform mat $\left\{\left(R(z, \varepsilon) \varphi_{i}, \varphi_{j}\right)\right\}$ of the matrixvalued measure $d M=\operatorname{mat}\left\{d\left(E_{\varepsilon}(\lambda) \varphi_{i}, \varphi_{j}\right)\right\}$ satisfies the hypotheses of Theorem 2 if $\zeta(\varepsilon)$ is taken as the origin. It follows that $\zeta(\varepsilon)$ is a point mass of $M$, and hence a point eigenvalue of $T_{\varepsilon}$.

Let $Q_{0}$ be the orthogonal projection on the eigenspace of $\zeta(\varepsilon)$ and $Q$ the orthogonal projection on $\mathscr{R}(V)$. Then the point mass of $M$ at $\zeta(\varepsilon)$ is the matrix of the operator $Q Q_{0} Q$ on $\mathscr{R}(V)$, and since this is the residue of mat $\left\{\left(R(z, \varepsilon) \varphi_{i}, \varphi_{j}\right)\right\}$, it has rank one. It follows that if $\psi_{1}$ and $\psi_{2}$ are in the range of $Q_{0}$, there exists a linear combination $\psi=a_{1} \psi_{1}+a_{2} \psi_{2}$ such that $Q Q_{0} Q \psi=0$. But then

$$
0=\left(Q_{0} Q \psi, Q \psi\right)=\left\|Q_{0} Q \psi\right\|^{2}
$$

so that $Q_{0} Q \psi=0$ and

$$
0=\left(Q \psi, Q_{0} \psi\right)=(Q \psi, \psi)=\|Q \psi\|^{2}
$$

Thus, if $\mathscr{C}_{0}$ is the orthogonal complement of the subspace spanned by $\psi$, then $\mathscr{L}_{0}$ reduces $T_{\varepsilon}$ (since $\psi$ is an eigenvector) and contains $\mathscr{R}(V)$ (since $Q \psi=0)$. Hence, $\mathscr{L}_{0}$ also reduces $T_{0}$, so that $\mathscr{C}_{0}=\mathscr{H}$ and $\psi=0$. Therefore $\psi_{1}$ and $\psi_{2}$ are linearly dependent, and $\zeta(\varepsilon)$ has simple multiplicity.

REMARK. Note that by analyticity of $\zeta(\varepsilon)$, either (a) $\operatorname{Im} \zeta(\varepsilon) \neq 0$ for all sufficiently small positive $\varepsilon$ or $(b) \operatorname{Im} \zeta(\varepsilon)=0$ for all sufficiently small positive $\varepsilon$.

5. Asymptotic spectral theory. In this section, an asymptotic formula for the spectral density matrix defined by

$$
E^{\prime}(\lambda, \varepsilon)=\operatorname{mat}\left\{d\left(E_{\varepsilon}(\lambda) \varphi_{i}, \varphi_{j}\right) / d \lambda\right\}
$$

is obtained. The result is valid near $\operatorname{Re} \zeta(\varepsilon)$ as $\varepsilon \rightarrow 0+$ in the case $\operatorname{Im} \zeta(\varepsilon) \neq 0$, and corresponds to the formula of Wigner and Weisskopf referred to by Friedrichs [4].

LEMMA 5.1. If we define

$$
\boldsymbol{R}_{+}(z, \varepsilon)=\operatorname{mat}\left\{\left(R(z, \varepsilon) \varphi_{i}, \varphi_{j}\right)_{+}\right\}
$$

then $(z-\zeta(\varepsilon)) \boldsymbol{R}_{+}(z, \varepsilon)$ is analytic in $z$ and $\varepsilon$ on $N \times\left\{\varepsilon:|\varepsilon|<\varepsilon_{1}\right\}$ for some sufficiently small positive $\varepsilon_{1}$.

Proof. Observe that $\varepsilon \Delta_{+}^{-1}(z, \varepsilon)=\lambda_{0}-z+\mathrm{O}(\varepsilon)$ uniformly on $N$. If $\left|\lambda_{0}-z\right|=\rho_{0}$, then $\left|\varepsilon \Delta_{+}^{-1}\right| \geqq\left(\rho_{0} / 2\right)$ for $\varepsilon$ small, and hence $\left|\Delta_{+}\right| \leqq\left(2 \varepsilon / \rho_{0}\right)$ uniformly on $\left|\lambda_{0}-z\right|=\rho_{0}$. From (4.1) and Theorem 1, $\boldsymbol{R}_{+}(z, \varepsilon)$ is 
analytic in $z$ for each $\varepsilon$, and by the above is analytic in $\varepsilon$ on $\left|z-\lambda_{0}\right|=$ $\rho_{0}$. Thus, we have by Cauchy's formula

$$
(z-\zeta(\varepsilon)) \boldsymbol{R}_{+}(z, \varepsilon)=(1 / 2 \pi i) \int_{\partial N}(\xi-z)^{-1}(\xi-\zeta(\varepsilon)) \boldsymbol{R}_{+}(\xi, \varepsilon) d \xi
$$

where the contour $\partial N$ has the positive orientation. By differentiation under the integral sign, we have analyticity in $\varepsilon$ in a neighborhood of $\varepsilon=0$.

Let $\zeta(\varepsilon)=\lambda_{\varepsilon}-i \Gamma_{c}$, so that $\Gamma_{\varepsilon} \geqq 0$, and let $|J|$ be the Lebesgue measure of $J$.

Theorem 4. Assume that $\operatorname{Im} \zeta(\varepsilon) \neq 0$, and let $\left\{J_{\varepsilon}: \varepsilon>0\right\}$ be an arbitrary family of open intervals containing $\lambda_{\varepsilon}$ and such that $\left|J_{\varepsilon}\right|=$ $\mathrm{O}\left(\Gamma_{\varepsilon}^{1 / 2}\right)$ as $\varepsilon \rightarrow 0+$. Then for $\lambda \in J_{\varepsilon}$

$$
E^{\prime}(\lambda, \varepsilon)=\left(\Gamma_{\varepsilon} / \pi\right)\left[\left(\lambda-\lambda_{\varepsilon}\right)^{2}+\Gamma_{\varepsilon}^{2}\right]^{-1} B(\varepsilon)+\mathrm{O}(1)
$$

as $\varepsilon \rightarrow 0+$, where $B(\varepsilon)$ is the real part of the residue of $\boldsymbol{R}_{+}(z, \varepsilon)$ at the pole $\zeta(\varepsilon)$. $B(\varepsilon)$ is analytic in $\varepsilon$ and $B(0)=\operatorname{mat}\left\{\left(P_{0} \varphi_{i}, \varphi_{j}\right)\right\}$.

Proof. By Lemma 5.1, we have

$$
\boldsymbol{R}_{+}(z, \varepsilon)=(z-\zeta(\varepsilon))^{-1} A(\varepsilon)+\mathrm{O}(1)
$$

as $\varepsilon \rightarrow 0+$, uniformly on $N$. Since

$$
A(\varepsilon)=(1 / 2 \pi i) \int_{\partial N} \boldsymbol{R}_{+}(z, \varepsilon) d z
$$

differentiation under the integral sign shows that $A(\varepsilon)$ is analytic near $\varepsilon=0$, and hence that $B(\varepsilon)$ is real analytic for real $\varepsilon$. Let

$$
A(\varepsilon)=B(\varepsilon)+i C(\varepsilon) \text {. }
$$

Then by (5.1) we have for $\lambda \in J_{s}$

$$
\begin{aligned}
\boldsymbol{E}^{\prime}(\lambda, \varepsilon)= & (1 / \pi) \operatorname{Im} \boldsymbol{R}_{+}(\lambda, \varepsilon) \\
= & \left(\Gamma_{\varepsilon} / \pi\right)\left[\left(\lambda-\lambda_{c}\right)^{2}+\Gamma_{\varepsilon}^{2}\right]^{-1} B(\varepsilon) \\
& +(1 / \pi)\left(\lambda-\lambda_{\varepsilon}\right)\left[\left(\lambda-\lambda_{\varepsilon}\right)^{2}+\Gamma_{\varepsilon}^{2}\right]^{-1} C(\varepsilon)+\mathrm{O}(1)
\end{aligned}
$$

as $\varepsilon \rightarrow 0+$. Observe now that $C(\varepsilon)=\mathrm{O}\left(\Gamma_{\varepsilon}^{1 / 2}\right)$; for if we note that $E^{\prime}(\lambda, \varepsilon) \geqq 0$ and set $\lambda=\lambda_{\varepsilon} \pm \Gamma_{s}^{1 / 2}$, then (5.2) implies that

$$
\pm \Gamma_{\varepsilon}^{1 / 2}\left[\Gamma_{\varepsilon}+\Gamma_{\varepsilon}^{2}\right]^{-1}(C(\varepsilon) x, x)+\mathrm{O}(1) \geqq 0
$$

for every $x \in \mathscr{R}(V)$. But if $\Gamma_{\varepsilon}^{-1 / 2}(C(\varepsilon) x, x)$ were unbounded as $\varepsilon \rightarrow 0+$, then since either sign of $\Gamma_{\varepsilon}$ can be taken, (5.3) could not hold. It now follows easily that the second term of (5.2) is $O(1)$ as $\varepsilon \rightarrow 0+$, 
which completes the proof.

6. Spectral concentration. Let $\left\{T_{\varepsilon}: \varepsilon \geqq 0\right\}$ be a family of selfadjoint operators. If $E_{\varepsilon}(\lambda)$ is the spectral resolution of $T_{\varepsilon}$, we define $E_{\mathrm{s}}[S]=\int_{S} d E_{\mathrm{\varepsilon}}(\lambda)$ for every Borel set $S$. Following [1], we say that the spectrum of $T_{\varepsilon}$ is concentrated at $\lambda_{0}$ to order $p$ as $\varepsilon \rightarrow 0+$ if and only if there exists a family $\left\{J_{\varepsilon}: \varepsilon>0\right\}$ of intervals such that $E_{\varepsilon}\left[J_{\varepsilon}\right] \rightarrow E_{0}\left[\left\{\lambda_{0}\right\}\right]$ strongly and $\left|J_{\varepsilon}\right|=O\left(\varepsilon^{p}\right)$ as $\varepsilon \rightarrow 0+$, where $\left|J_{\varepsilon}\right|$ is the Lebesgue measure of $J_{\varepsilon}$.

THeOREM 5. Let $\left\{J_{\varepsilon}: \varepsilon>0\right\}$ be an arbitrary family of intervals, symmetric about $\lambda_{\varepsilon}$, and such that $\left|J_{\varepsilon}\right|=\mathrm{O}\left(\Gamma_{\varepsilon}^{1 / 2}\right)$ as $\varepsilon \rightarrow 0+$. Assume that $\operatorname{Im} \zeta(\varepsilon) \neq 0$. Then

(a) If $\beta=\lim \left|J_{\varepsilon}\right| / 2 \Gamma_{\varepsilon}$ exists as

$$
\varepsilon \rightarrow 0+, 0 \leqq \beta \leqq \infty,
$$

then $E_{\varepsilon}\left[J_{\varepsilon}\right] \rightarrow(2 / \pi)$ arctan $(\beta) P_{0}$ weakly as $\varepsilon \rightarrow 0+$

(b) $E_{\varepsilon}\left[J_{\varepsilon}\right] \rightarrow P_{0}$ strongly if and only if $\lim \left|J_{\varepsilon}\right| / \Gamma_{\varepsilon}=\infty$.

CoRollary 6.1. Let $\Gamma_{\varepsilon}=\mathrm{O}\left(\varepsilon^{q}\right)$ as $\varepsilon \rightarrow 0+$. Then the spectrum of $T_{\varepsilon}$ is concentrated at $\lambda_{0}$ to order $p$ for $0 \leqq p<q$, but not for $p \geqq q$.

Proof of Theorem 5. If we recall that $(B(\varepsilon) x, y) \rightarrow\left(P_{0} x, y\right)$ as $\varepsilon \rightarrow 0+$ for every $x, y \in \mathscr{R}(V)$, the proof is essentially the same as the proof of Theorem 4 of [5]. Only a few simple changes need to be made to compensate for the fact that in [5], $\Gamma_{\varepsilon}=\mathrm{O}\left(\varepsilon^{2}\right)$.

REMARK. In defining concentration of order $p$, the authors of [1] permit $\left\{J_{\varepsilon}\right\}$ to be Borel sets. The distinction is relevant for eigenvalues $\lambda_{0}$ of multiplicity greater than one, but not for simple multiplicity - at least for isolated eigenvalues. We have therefore chosen the simpler definition.

7. Perturbation series. One of the classical problems of perturbation theory (discussed, for example, by Frierdrichs [4] and Kato [8, Chap. III]) is the interpretation of the formal perturbation series in cases where it exists (at least to a certain number of terms), but there is no corresponding perturbed eigenvalue. This is usually done in terms of spectral concentration (see $[1,8,10]$ where further references are given). In the preceding section, we have related spectral concentration to the position of the pole $\zeta(\varepsilon)$, and we shall now discuss the connection between $\zeta(\varepsilon)$ and the formal series. Under some rea- 
sonable nondegeneracy conditions, we obtain rather instructive results, which involve the order of vanishing of the unperturbed spectral density at $\lambda_{0}$. To be precise, we shall prove the following theorem:

THEOREM 6. Let $\nu$ be the order of vanishing of the matrixvalued function mat $\left\{d\left(E_{0}(\lambda) P \varphi_{i}, \varphi_{j}\right) / d \lambda\right\}$ at $\lambda_{0}$, and assume that

$$
d\left(E_{0}(\lambda) P V \psi_{0}, \psi_{0}\right) / d \lambda
$$

vanishes at $\lambda_{0}$ of order exactly $\nu$. Let

$$
\zeta(\varepsilon)=\sum_{n=0}^{\infty} a_{n} \varepsilon^{n}
$$

be the series expansion of $\zeta(\varepsilon)$, and let

$$
\zeta_{0}(\varepsilon)=\lambda_{0}+\lambda_{p} \varepsilon^{p}+\mathrm{O}\left(\varepsilon^{p+1}\right)
$$

where $\lambda_{p} \neq 0, p \geqq 1$ and $\zeta_{0}(\varepsilon)$ is the quantity defined in Lemma 7.2 below. Then

(a) The first nonreal coefficient in (7.1) is $a_{2+\nu p}$ and we have

$$
\Gamma_{\varepsilon}=+\varepsilon^{2+\nu p} \lambda_{p}^{\nu} \gamma\left(\lambda_{0}\right)+\mathrm{O}\left(\varepsilon^{\nu p+3}\right)
$$

where

$$
\gamma(\lambda)=\pi\left(\lambda-\lambda_{0}\right)^{-\nu} d\left(E_{0}(\lambda) P V \psi_{0}, V \psi_{0}\right) / d \lambda .
$$

(b) The formal perturbation series exists up through terms of order $\nu p+1$ and coincides with $\sum_{n=0}^{\nu p+1} a_{n} \varepsilon^{n}$.

For the proof, we shall require two lemmas.

LEMma 7.1. Let $C$ be the contour consisting of the real axis outside of $G=\left(\lambda_{0}-\rho_{0}, \lambda_{0}+\rho_{0}\right)$, together with the lower boundary of $N$, all positively oriented. Then for any $n \geqq 0$ and $\operatorname{Im} z>0$, we have

$$
W_{a}(z, \varepsilon)=I+\varepsilon Q_{n}(z)+\varepsilon\left(z-\lambda_{0}\right)^{n} D_{n}(z)
$$

where $Q_{n}(z)$ is the matrix polynomial $\sum_{k=0}^{n-1} Q^{(k)}\left(z-\lambda_{0}\right)^{k}$, with coefficients

$$
Q^{(k)}=\operatorname{mat}\left\{c_{j} \int_{C}\left(\lambda-\lambda_{0}\right)^{-k-1} d\left(E_{0}(\lambda) P \varphi_{i}, \varphi_{j}\right)\right\}
$$

and

$$
D_{n}(z)=\operatorname{mat}\left\{c_{j} \int_{C}\left(\lambda-\lambda_{0}\right)^{-n}(\lambda-z)^{-1} d\left(E_{0}(\lambda) P \varphi_{i}, \varphi_{j}\right)\right\} .
$$


REMARKS. (1) The meaning of the rather cryptic integrals on the right sides of (7.5) and (7.6) is the following. The integral has its usual meaning over $\left|\lambda-\lambda_{0}\right| \geqq \rho_{0}$, while on the lower part of $\partial N$, $d\left(E_{0}(\lambda) P \varphi_{i}, \varphi_{j}\right)$ is to be replaced by

$$
(1 / 2 \pi i)\left[\left(R_{0}(\lambda) P \varphi_{i}, \varphi_{j}\right)_{+}-\left(R_{0}(\lambda) P \varphi_{i}, \varphi_{j}\right)_{-}\right] d \lambda .
$$

The coefficient of $d \lambda$ in (7.7) is simply the analytic continuation of $d\left(E_{0}(\lambda) \varphi_{i}, \varphi_{i}\right) / d \lambda$ from $G$ to $N$.

(2) Note that since $D_{n}(z)$ is well defined and analytic on the interior of $N$, and $Q_{n}(z)$ is a polynomial, (7.4) holds on the interior of $N$, provided that $W_{a}(z, \varepsilon)$ is replaced by $\left[W_{a}(z, \varepsilon)\right]_{+}$on the left. A similar formula can be obtained for $\left[W_{a}(z, \varepsilon)\right]_{\text {- simply by making } C}$ traverse the (negatively oriented) upper half of the boundary of $N$ rather than the lower half.

Proof of Lemma 7.1. Fix $z$ with $\operatorname{Im} z>0$ and note the finite binomial expansion

$$
\begin{aligned}
(\lambda-z)^{-1}= & \left(\lambda-\lambda_{0}\right)^{-1} \sum_{k=0}^{n-1}\left\{\left(z-\lambda_{0}\right)^{k}\left(\lambda-\lambda_{0}\right)^{-k}\right. \\
& \left.+\left(z-\lambda_{0}\right)^{n}\left(\lambda-\lambda_{0}\right)^{-n}(\lambda-z)^{-1}\right\} .
\end{aligned}
$$

Integrate (7.8) over $C$ with respect to the matrix measure

$$
\operatorname{mat}\left\{\varepsilon c_{j} d\left(E_{0}(\lambda) P \varphi_{i}, \varphi_{j}\right)\right\}
$$

(see Remark (1) above). On the right, we obtain

$$
\varepsilon Q_{n}(z)+\varepsilon\left(z-\lambda_{0}\right)^{n} D_{n}(z)
$$

and on the left

$$
\operatorname{mat}\left\{\varepsilon c_{j} \int_{C}(\lambda-z)^{-1} d\left(E_{0}(\lambda) P \varphi_{i}, \varphi_{j}\right)\right\} \text {. }
$$

For $\operatorname{Im} z>0$, the integrand of (7.9) is analytic between $C$ and the real axis, and we may therefore deform the contour $C$ in (7.9) to the real axis. When this is done, we find that (7.9) is equal to $W_{a}(z, \varepsilon)-I$, which yields the result.

Lemma 7.2. (a) For sufficiently small $\varepsilon$, there exists in $N a$ unique solution $z=\zeta_{0}(\varepsilon)$ of the equation

$$
z=\lambda_{0}+\varepsilon\left(\left[I+\varepsilon Q_{\nu}(z)\right]^{-1} V \psi_{0}, \psi_{0}\right)
$$

$\zeta_{0}(\varepsilon)$ is analytic in $\varepsilon$ at $\varepsilon=0$, and $\zeta_{0}(0)=\lambda_{0}$. 
Moreover, (b) $\zeta_{0}(\varepsilon)$ is real for real $\varepsilon$ and

(c) coincides with the formal perturbation series up to and including terms of order $\varepsilon^{\nu p+1}$.

Proof of parts $(a)$ and $(b)$. The equation (7.10) is sufficiently like (2.1) that part (a) can be proved just as Theorem 1. For part (b), observe that since mat $\left\{d\left(E_{0}(\lambda) P \varphi_{i}, \varphi_{j}\right) / d \lambda\right\}$ vanishes at $\lambda_{0}$ of order $\nu$, the contour of integration in (7.5) can be deformed back to the real axis to obtain

$$
\begin{aligned}
Q^{(k)} & =\operatorname{mat}\left\{c_{j} \int_{-\infty}^{+\infty}\left(\lambda-\lambda_{0}\right)^{-k-1} d\left(E_{0}(\lambda) P \varphi_{i}, \varphi_{j}\right)\right\} \\
& =\operatorname{mat}\left\{c_{j}\left(S^{k+1} \varphi_{i}, \varphi_{j}\right)\right\}
\end{aligned}
$$

where $S$ is the reduced resolvent (cf. [1] and [8]), defined by

$$
\begin{aligned}
& S=\left(T_{0} P-\lambda_{0} P\right)^{-1} \text { on } P \mathscr{H} \\
& =0 \quad \text { on } P_{0} \mathscr{H} \text {. }
\end{aligned}
$$

Now $S$ is self-adjoint, and hence the matrix polynomial

$$
S(z)=\sum_{k=0}^{n-1} \operatorname{mat}\left\{\left(S^{k+1} \varphi_{i}, \varphi_{j}\right)\right\} z^{k}
$$

is Hermitian for real $z$. It follows by a simple calculation that the matrix

$$
\left[I+\varepsilon Q_{\nu}(z)\right]^{-1} V=[I+\varepsilon V S(z)]^{-1} V
$$

is Hermitian, and hence that the right side of (7.10) is real for real $z$ in $N$. However, it now follows by the Schwartz reflection principle that the complex conjugate of any solution of (7.10) is also a solution. Since $\zeta_{0}(\varepsilon)$ is unique, it is therefore real.

For the proof of (c), we shall require the following.

Proposition. Let $F(z, \varepsilon)$ and $F_{0}(z, \varepsilon)$ be analytic in $z$ on the unit disc, for each fixed $\varepsilon, 0 \leqq \varepsilon \leqq \varepsilon_{2}$. Let $d F / d z$ be bounded on the unit disc uniformly in $\varepsilon$, and suppose that for some $m \geqq 0$

$$
F(z, \varepsilon)-F_{0}(z, \varepsilon)=\mathrm{O}\left(\varepsilon z^{m}\right)
$$

uniformly in $\varepsilon$. Then if there exist unique solutions $z(\varepsilon)$ and $z_{0}(\varepsilon)$ of the equations $z=\varepsilon F(z, \varepsilon)$ and $z_{0}=\left(z_{0}, \varepsilon\right)$ in the unit disc, we have

$$
z(\varepsilon)-z_{0}(\varepsilon)=\mathrm{O}\left(\varepsilon^{2} z_{0}^{m}\right)
$$

as $\varepsilon \rightarrow 0+$.

Proof. We have, uniformly in $\varepsilon$, 


$$
\begin{aligned}
z-z_{0} & =\varepsilon F(z, \varepsilon)-\varepsilon F_{0}\left(z_{0}, \varepsilon\right) \\
& =\varepsilon F(z, \varepsilon)-\varepsilon F\left(z_{0}, \varepsilon\right)+O\left(\varepsilon^{2} z_{0}^{m}\right) \\
& =\varepsilon O\left(z-z_{0}\right)+O\left(\varepsilon^{2} z_{0}^{m}\right)
\end{aligned}
$$

where the final equality uses uniform boundedness of $d F / d z$. Hence

$$
\left(z-z_{0}\right)(1+\mathrm{O}(\varepsilon))=\mathrm{O}\left(\varepsilon^{2} z_{0}^{m}\right)
$$

which implies (7.11) as $\varepsilon \rightarrow 0+$.

Proof of part (c). Consider first the case where $\lambda_{0}$ is an isolated eigenvalue of $T_{0}$, (see [8, Chap. VII]) so that $d E_{0}(\lambda) P / d \lambda=0$ on a neighborhood of $G$. In this case, there is a perturbed eigenvalue $\zeta(\varepsilon)$ which satisfies the equation

$$
z=\lambda_{0}+\varepsilon\left([I+\varepsilon K(z)]^{-1} V \psi_{0}, \psi_{0}\right)
$$

where $K(z)$ is the matrix mat $\left\{c_{j}\left(R_{0}(z) P \varphi_{i}, \varphi_{j}\right)\right\}$. Now as an operator on $\mathscr{H}, R_{0}(z) P$ is analytic on a neighborhood of $N$, so that

$$
V R_{0}(z) P=V \sum_{k=0}^{\infty}\left[R_{0}(z) P\right]^{k+1}\left(z-\lambda_{0}\right)^{k}
$$

for $z$ in $N$, from which it is immediate that

$$
K(z)=Q_{n}(z)+\mathrm{O}\left(\left(z-\lambda_{0}\right)^{n}\right)
$$

for every $n \geqq 0$. By the above proposition, taking $\lambda_{0}$ as the origin, we may compare the solutions of (7.10) and (7.12) for $n=\nu$ to obtain that

$$
\zeta(\varepsilon)-\zeta_{0}(\varepsilon)=O\left(\varepsilon^{p \nu+2}\right)
$$

where $\zeta_{0}$ corresponds to $Q_{\nu}$, and $p$ is given by (7.2).

Now the formal perturbation series may be defined as the series whose terms are formally the same as those of $\zeta(\varepsilon)$ in the isolated case, leaving aside all questions of convergence and existence of terms. Moreover, the coefficients of $\zeta_{0}(\varepsilon)$ obtained from (7.10) are formally the same in all cases, provided only that the terms of $Q_{\nu}(z)$ exist. Hence, even in the present case, the equation (7.13) must hold in the formal sense, which is all that is required. This rather devious proof avoids a direct calculation of the formal series, a task which sceptics are invited to perform.

Proof of Theorem 6. Applying the formula

$$
(I+a+b)^{-1}=(I+a)^{-1}-(I+a)^{-1} b(I+a)^{-1}+\mathrm{O}\left(b^{2}\right)
$$

to (7.4) with $n=\nu$, we obtain 


$$
\begin{aligned}
W_{a}^{-1}(z, \varepsilon)= & {\left[I+\varepsilon Q_{\nu}(z)\right]^{-1} } \\
& -\left[I+\varepsilon Q_{\nu}(z)\right]^{-1} \varepsilon\left(z-\lambda_{0}\right)^{\nu} D_{\nu}(z)\left[I+\varepsilon Q_{\nu}(z)\right]^{-1} \\
& +O\left(\varepsilon^{2}\left(z-\lambda_{0}\right)^{2 \nu}\right) \\
= & {\left[I+\varepsilon Q_{\nu}(z)\right]^{-1}-\varepsilon\left(z-\lambda_{0}\right)^{\nu} D_{\nu}(z)+O\left(\varepsilon^{2}\left(z-\lambda_{0}\right)^{\nu}\right) . }
\end{aligned}
$$

Define $\zeta_{1}(\varepsilon)=\zeta(\varepsilon)-\zeta_{0}(\varepsilon)$. Since $\zeta(\varepsilon)$ satisfies (2.1), we obtain from (7.14)

$$
\begin{aligned}
\left(\zeta_{0}(\varepsilon)-\lambda_{0}\right) & +\zeta_{1}(\varepsilon)=\varepsilon\left(\left[I+\varepsilon Q_{\nu}(\zeta(\varepsilon))\right]^{-1} V \psi_{0}, \psi_{0}\right) \\
& -\varepsilon^{2}\left(\zeta(\varepsilon)-\lambda_{0}\right)^{\nu}\left(D_{\nu}(\zeta(\varepsilon)) V \psi_{0}, \psi_{0}\right)+O\left(\varepsilon^{3}\left(\zeta(\varepsilon)-\lambda_{0}\right)^{\nu}\right) .
\end{aligned}
$$

Now $\zeta(\varepsilon)$ can be replaced by $\zeta_{0}(\varepsilon)$ in the first term on the right side of (7.15), to within a term which is $O\left(\varepsilon \zeta_{1}(\varepsilon)\right)$. By definition of $\zeta_{0}(\varepsilon)$, the resulting term will exactly cancel $\zeta_{0}(\varepsilon)-\lambda_{0}$ on the left side, yielding

$$
\begin{aligned}
\zeta_{1}(\varepsilon) & +\mathrm{O}\left(\varepsilon \zeta_{1}(\varepsilon)\right)=-\varepsilon^{2}\left(\zeta(\varepsilon)-\lambda_{0}\right)^{2}\left(D_{\nu}(\zeta(\varepsilon)) V \psi_{0}, \psi_{0}\right) \\
& +\mathrm{O}\left(\varepsilon^{3}\left(\zeta(\varepsilon)-\lambda_{0}\right)^{2}\right) .
\end{aligned}
$$

The second term on the left side may clearly be dropped as $\varepsilon \rightarrow 0+$. Observe now that if $\nu>0$, then

$$
\left(\zeta(\varepsilon)-\lambda_{0}\right)^{\nu}=\left(\zeta_{0}(\varepsilon)-\lambda_{0}\right)^{\nu}+\left(\zeta_{0}(\varepsilon)-\lambda_{0}\right)^{\nu-1} \mathrm{O}\left(\zeta_{1}(\varepsilon)\right) .
$$

If this expression is inserted into the two terms on the right side of (7.16), it is seen in a similar way that of the four resulting terms, the two involving $\zeta_{1}(\varepsilon)$ may both be dropped. We therefore obtain

$$
\zeta_{1}(\varepsilon)=-\varepsilon^{2}\left(\zeta_{0}(\varepsilon)-\lambda_{0}\right)^{\nu}\left(D_{\nu}(\zeta(\varepsilon)) V \psi_{0}, \psi_{0}\right)+\mathrm{O}\left(\varepsilon^{3}\left(\zeta_{0}(\varepsilon)-\lambda_{0}\right)^{\nu}\right)
$$

for $\nu>0$; however, it is trivial from (7.16) that (7.17) also holds for $\nu=0$.

Now, since $\zeta_{0}(\varepsilon)$ is real for real $\varepsilon$,

$$
\Gamma_{\varepsilon}=-\operatorname{Im} \zeta(\varepsilon)=-\operatorname{Im} \zeta_{1}(\varepsilon) .
$$

Taking the imaginary part of (7.17) and using (7.2), we have that

$$
\Gamma_{\varepsilon}=\varepsilon^{\nu p+2} \lambda_{p}^{\nu} \operatorname{Im}\left(D_{\nu}(\zeta(\varepsilon)) V \psi_{0}, \psi_{0}\right)+\mathrm{O}\left(\varepsilon^{\nu p+3}\right) .
$$

Since $D_{\nu}(z)$ is analytic, $\zeta(\varepsilon)$ may be replaced by $\lambda_{0}$ on the right side of (7.18), with the remainder being absorbed in the second term. However, by (7.6), we have

$$
\operatorname{Im}\left(D_{\nu}\left(\lambda_{0}\right) V \psi_{0}, \psi_{0}\right)=\lim _{z \rightarrow \lambda_{0}+i_{0}} \int_{-\infty}^{+\infty}\left[\operatorname{Im}(\lambda-z)^{-1}\right]\left(\lambda-\lambda_{0}\right)^{-\nu} d\left(E_{0}(\lambda) P V \psi_{0}, V \psi_{0}\right)
$$

which is just a Poisson integral, convergent to $\gamma\left(\lambda_{0}\right)$. (7.3) now follows from (7.18), and the remainder of part (a) follows from (7.3). For part (b), observe that by (7.17) 


$$
\zeta(\varepsilon)-\zeta_{0}(\varepsilon)=O\left(\varepsilon^{\nu p+2}\right)
$$

and that, by Lemma $7.2, \zeta_{0}(\varepsilon)$ differs from the formal series by terms which are $\mathrm{O}\left(\varepsilon^{\nu p+2}\right)$.

8. Examples. We shall first consider the classical example of Friedrichs $\left[4, \S^{\prime s} 6-8\right.$ and 10]. Let $\mathscr{F}$ be a closed interval and $\mathscr{H}=$ $L_{2}(\mathscr{J}) \oplus C$ where $C$ is the one-dimensional space of complex numbers. Let

$$
T_{0}[u(\lambda), \xi]=\left[\lambda u(\lambda), \lambda_{0} \xi\right]
$$

where $\lambda_{0}$ is in the interior of $\mathscr{F}_{0} T_{0}$ is self-adjoint with spectrum $\mathscr{I}$ and has absolutely continuous spectral measure, except for an eigenvalue $\lambda_{0}$ of simple multiplicity with eigenvector $\psi_{0}=[0,1]$. Let

$$
V=(\cdot, f) \psi_{0}+\left(\cdot, \psi_{0}\right) f
$$

where $f=[f(\lambda), 0]$. We shall assume that (a) $f(\lambda)$ is Holder continuous on $(-\infty,+\infty)$ and has its support in $\mathscr{F}$, (b) $f(\lambda)$ does not vanish on the interior of $\mathscr{J}$ and (c)

$$
\int_{\Omega}|f(\lambda)|^{2} d \lambda=1
$$

$V$ is self-adjoint of rank two, and $\mathscr{R}(V)$ has the orthonormal basis $\left\{f, \psi_{0}\right\}$. We shall compute matrices on $\mathscr{R}(V)$ with respect to this basis; the fact that $V$ is not diagonal is of no importance.

Let

$$
T_{\varepsilon}=T_{0}+\varepsilon V \text {. }
$$

For the matrix of $I+\varepsilon V R_{0}(z)$ on $\mathscr{R}(V)$ with respect to the basis $\left\{f, \psi_{0}\right\}$ we obtain

$$
W(z, \varepsilon)=\left|\begin{array}{cc}
1 & \varepsilon\left(\lambda_{0}-z\right)^{-1} \\
\varepsilon F(z) & 1
\end{array}\right|
$$

where

$$
F(z)=\int_{\mathscr{\Omega}}|f(\lambda)|^{2}(\lambda-z)^{-1} d \lambda
$$

The inverse matrix is

$$
W^{-1}(z, \varepsilon)=\left[\lambda_{0}-z-\varepsilon^{2} F(z)\right]^{-1}\left|\begin{array}{cc}
\left(\lambda_{0}-z\right) & -\varepsilon \\
-\varepsilon\left(\lambda_{0}-z\right) F(z) & \left(\lambda_{0}-z\right)
\end{array}\right| .
$$

Since $f(\lambda)$ is Holder continuous, $F(z)$ is continuous up to the real axis and

$$
\operatorname{Im} F(\lambda+i 0)=\pi|f(\lambda)|^{2}
$$


By assumption (b), this quantity does not vanish on the interior of $\mathscr{F}$. Hence, $T_{\varepsilon}$ is absolutely continuous on the interior of $\mathscr{F}$, and the eigenvalue $\lambda_{0}$ disappears under the perturbation $\varepsilon V$.

Now if $|f(\lambda)|^{2}$ is analytic near $\lambda_{0}$, then by Theorem 2 and (8.3), $F(z)$ will have a continuation $F_{+}(z)$. It is clear from (8.2) that the virtual poles are solutions of

$$
\lambda_{0}-z-\varepsilon^{2} F_{+}(z)=0 .
$$

In the following three examples, $\mathscr{I}=(-\infty,+\infty)$ and $\lambda_{0}=0$.

EXAMPLE 8.1. Let

$$
|f(\lambda)|^{2}=(1 / \pi)\left(1+\lambda^{2}\right)^{-1} .
$$

Then for $\operatorname{Im} z>0$

$$
F(z)=-(z+i)^{-1}
$$

so that by continuation

$$
F_{+}(z)=-(z+i)^{-1}
$$

for all $z$. (8.4) therefore becomes

$$
-z+\varepsilon^{2}(z+i)^{-1}=0
$$

which, by the quadratic formula, has one solution $-i+O\left(\varepsilon^{2}\right)$ and another

$$
z(\varepsilon)=-i \varepsilon^{2}+\mathrm{O}\left(\varepsilon^{4}\right) .
$$

In order to apply (7.3), note that $\nu=0$ and hence by (8.3) that

$$
\gamma(\lambda)=\pi d\left(E_{0}(\lambda) P V \psi_{0}, V \psi_{0}\right) / d \lambda=\pi|f(\lambda)|^{2} .
$$

By (8.5), this gives

$$
\Gamma(\varepsilon)=\varepsilon^{2}+\mathrm{O}\left(\varepsilon^{3}\right)
$$

which agrees with the actual value

$$
\Gamma(\varepsilon)=\varepsilon^{2}+\mathrm{O}\left(\varepsilon^{4}\right)
$$

obtained from (8.6).

EXAMPLE 8.2. If, instead of (8.5), we choose

$$
|f(\lambda)|^{2}=(2 / \pi) \lambda^{2}\left(1+\lambda^{2}\right)^{-2} .
$$

Then 


$$
F_{+}(z)=-z(z+i)^{-2}
$$

and (8.4) becomes

$$
-z+\varepsilon^{2} z(z+i)^{-2}=0
$$

which has the exact solutions

$$
z=0 \text { and } z=-i \pm \varepsilon^{2} \text {. }
$$

Therefore, this perturbation does not remove the eigenvalue $\lambda_{0}=0$. Theorem 6 does not apply; for although the condition involving $\nu$ is satisfied, a computation shows that, in the basis $\left\{f, \psi_{0}\right\}$,

$$
Q_{\nu}(z)=Q_{2}(z)=\left|\begin{array}{ll}
z & 0 \\
0 & 0
\end{array}\right|
$$

from which it follows that $\zeta_{0}(\varepsilon) \equiv 0$.

EXAMPLE 8.3. In order to illustrate (7.3) when $\nu \neq 0$, we consider a slightly different perturbation. Let

$$
V_{1}[u(\lambda), \xi]=\left[0, \lambda_{1} \xi\right]
$$

where $\lambda_{1}$ is real and nonzero, and define

$$
T_{\varepsilon}=T_{0}+\varepsilon V+\varepsilon V_{1} \text {. }
$$

Then $\mathscr{R}\left(V+V_{1}\right)=\mathscr{R}(V)$, and (8.1) acquires an additional term to become

$$
W(z, \varepsilon)=\left|\begin{array}{cr}
1 & \varepsilon\left(\lambda_{0}-z\right)^{-1} \\
\varepsilon F(z) & 1+\varepsilon \lambda_{1}\left(\lambda_{0}-z\right)^{-1}
\end{array}\right|
$$

The inverse is

$$
W^{-1}(z, \varepsilon)=\left[\lambda_{0}+\varepsilon \lambda_{1}-z-\varepsilon^{2} F(z)\right]^{-1}\left|\begin{array}{cc}
\left(\lambda_{0}+\varepsilon \lambda_{1}-z\right) & -\varepsilon \\
-\varepsilon\left(\lambda_{0}-z\right) F(z) & \left(\lambda_{0}-z\right)
\end{array}\right|
$$

and the equation for virtual poles becomes

$$
\lambda_{0}+\varepsilon \lambda_{1}-z+\varepsilon^{2} F(z)=0 .
$$

If $|f(\lambda)|^{2}$ is again given by (8.8), this equation becomes

$$
\left(-z+\varepsilon \lambda_{1}\right)(z+i)^{2}+\varepsilon^{2} z=0 .
$$

By successive approximations, the relevant root is

$$
z(\varepsilon)=\varepsilon \lambda_{1}-\varepsilon^{3} \lambda_{1}-2 \varepsilon^{4} \lambda_{1}^{2} i+\mathrm{O}\left(\varepsilon^{5}\right)
$$

so that 


$$
\Gamma(\varepsilon)=2 \lambda_{1}^{2} \varepsilon^{4}+\mathrm{O}\left(\varepsilon^{5}\right)
$$

by actual computation. To apply (7.3), note that $\nu=2$ and $\zeta_{0}(\varepsilon)=$ $\varepsilon \lambda_{1}+O\left(\varepsilon^{2}\right)$ so that $p=1$. Hence,

$$
\Gamma(\varepsilon)=\varepsilon^{4} \lambda_{1}^{2} \gamma(0)+\mathrm{O}\left(\varepsilon^{5}\right)
$$

where, by (8.7)

$$
\gamma(\lambda)=\lambda^{-2} \pi|f(\lambda)|^{2}=2\left(1+\lambda^{2}\right)^{-2} .
$$

Thus $\gamma(0)=2$, and (8.11) agrees with (8.10).

\section{BIBLIOGRAPHY}

1. C. C. Conley, and P. A. Rejto. "Spectral concentration, II-General Theory," Perturbation theory and its applications in Quantum mechanics ed. C. H. Wilcox Wiley, 1966.

2. C. L. Dolph, Recent developments in some non-self-adjoint problems of mathematical physics, Bull. Amer Math. Soc. 67 (1961), 1-69.

3. M. A. Evgrofov, Analytic functions, Saunders, Philadelphia, 1966. (Translated from Russian, Moscow, 1965.)

4. K. O. Friedrichs, On the perturbation of continuous spectra, Comm. Pure Appl. Math. 1 (1948) 361-406.

5. J. S. Howland, Perturbation of embedded eigenvalues by operators of finite rank, J. Math. Anal. Appl. 23 (1968), 575-584.

6. T. Kato, On the convergence of the perturbation method, J. Fac. Sci. Univ. Tokyo. 6 (1951), 145-226.

7. - On finite dimensional perturbations of self-adjoint operators, J. Math. Soc. Japan 9 (1957), 239-249.

8. — Perturbation theory for linear operators, Springer, 1966.

9. S. T. Kuroda, Finite-dimensional perturbation and a representation of scattering operator, Pacific J. Math. 13 (1963), 1305-1318.

10. J. B. McLeod, "Spectral concentration I-the one-dimensional Schroedinger operator", Perturbation theory and its applications in Quantum mechanics, ed. C. H. Wilcox, Wiley, 1966.

Received June 13, 1968.

UNIVERSITY OF VIRGINIA

Charlottesville, Virginia 


\section{PACIFIC JOURNAL OF MATHEMATICS}

\section{EDITORS}

H. ROYDEN

Stanford University

Stanford, California

\author{
R. R. Phelps \\ University of Washington \\ Seattle, Washington 98105
}

J. DugundJI

Department of Mathematics

University of Southern California

Los Angeles, California 90007

RICHARD ARENS

University of California

Los Angeles, California 90024

\section{ASSOCIATE EDITORS}

E. F. BECKenbaCH

B. H. NEUMANN

F. WOLF

K. YoSHIDA

\section{SUPPORTING INSTITUTIONS}

\author{
UNIVERSITY OF BRITISH COLUMBIA \\ CALIFORNIA INSTITUTE OF TECHNOLOGY \\ UNIVERSITY OF CALIFORNIA \\ MONTANA STATE UNIVERSITY \\ UNIVERSITY OF NEVADA \\ NEW MEXICO STATE UNIVERSITY \\ OREGON STATE UNIVERSITY \\ UNIVERSITY OF OREGON \\ OSAKA UNIVERSITY \\ UNIVERSITY OF SOUTHERN CALIFORNIA
}

\author{
STANFORD UNIVERSITY \\ UNIVERSITY OF TOKYO \\ UNIVERSITY OF UTAH \\ WASHINGTON STATE UNIVERSITY \\ UNIVERSITY OF WASHINGTON \\ $\stackrel{*}{*} \stackrel{*}{*} \stackrel{*}{*}$ AMERICAN MATHEMATICAL SOCIETY \\ CHEVRON RESEARCH CORPORATION \\ TRW SYSTEMS \\ NAVAL WEAPONS CENTER
}

The Supporting Institutions listed above contribute to the cost of publication of this Journal, but they are not owners or publishers and have no responsibility for its content or policies.

Mathematical papers intended for publication in the Pacific Journal of Mathematics should be in typed form or offset-reproduced, double spaced with large margins. Underline Greek letters in red, German in green, and script in blue. The first paragraph or two must be capable of being used separately as a synopsis of the entire paper. It should not contain references to the bibliography. Manuscripts, in duplicate if possible, may be sent to any one of the four editors. Please classify according to the scheme of Math. Rev. 36, 1539-1546. All other communications to the editors should be addressed to the managing editor, Richard Arens, University of California, Los Angeles, California, 90024.

50 reprints are provided free for each article; additional copies may be obtained at cost in multiples of 50 .

The Pacific Journal of Mathematics is published monthly. Effective with Volume 16 the price per volume (3 numbers) is $\$ 8.00$; single issues, $\$ 3.00$. Special price for current issues to individual faculty members of supporting institutions and to individual members of the American Mathematical Society: $\$ 4.00$ per volume; single issues $\$ 1.50$. Back numbers are available.

Subscriptions, orders for back numbers, and changes of address should be sent to Pacific Journal of Mathematics, 103 Highland Boulevard, Berkeley, California, 94708.

PUBLISHED BY PACIFIC JOURNAL OF MATHEMATICS, A NON-PROFIT CORPORATION

Printed at Kokusai Bunken Insatsusha (International Academic Printing Co., Ltd.), 7-17, Fujimi 2-chome, Chiyoda-ku, Tokyo, Japan. 


\section{Pacific Journal of Mathematics \\ Vol. 29, No. $3 \quad$ July, 1969}

Herbert James Alexander, Extending bounded holomorphic functions from certain subvarieties of a polydisc ...................... 485

Edward T. Cline, On an embedding property of generalized Carter

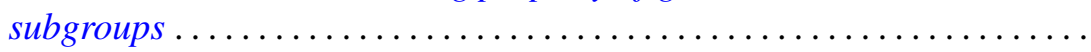

Roger Cuppens, On the decomposition of infinitely divisible characteristic functions with continuous Poisson spectrum. II ...............

William Richard Emerson, Translation kernels on discrete Abelian

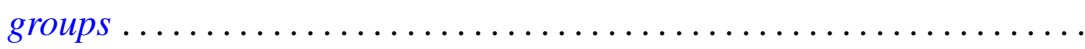

Robert William Gilmer, Jr., Power series rings over a Krull domain ....... 543

Julien O. Hennefeld, The Arens products and an imbedding theorem ...... 551

James Secord Howland, Embedded eigenvalues and virtual poles ........ 565

Bruce Ansgar Jensen, Infinite semigroups whose non-trivial homomorphs are all isomorphic .............................. 583

Michael Joseph Kascic, Jr., Polynomials in linear relations. II .......... 593

J. Gopala Krishna, Maximum term of a power series in one and several

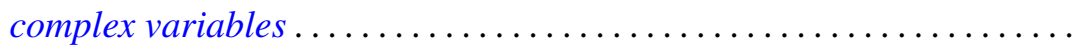

Renu Chakravarti Laskar, Eigenvalues of the adjacency matrix of cubic lattice graphs ...................................

Thomas Anthony Mc Cullough, Rational approximation on certain plane

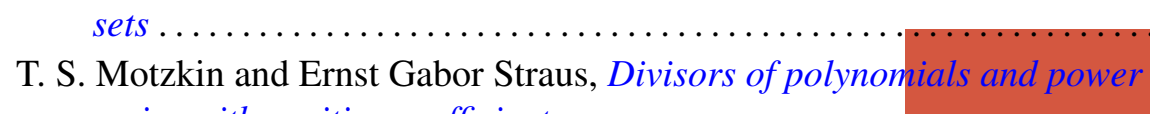
series with positive coefficients .

Graciano de Oliveira, Matrices with prescribed characteristic polynomial and a prescribed submatrix.

Graciano de Oliveira, Matrices with prescribed characteristic polynomial and a prescribed submatrix. II .

Donald Steven Passman, Exceptional 3/2-transitive permutation groups .................................

Grigorios Tsagas, A special deformation of the metric with no negative sectional curvature of a Riemannian space............

Joseph Zaks, Trivially extending decompositions of $E^{n}$ 